\title{
$\mathrm{LiMn}_{2} \mathrm{O}_{4}$ 尖晶石氧化物的低指数表面结构优化及表面能的 第一性原理研究
}

\author{
陆远 王继芬* 谢华清 \\ (上海第二工业大学理学院 上海先进热功能材料工程技术研究中心 上海 201209)
}

\begin{abstract}
摘要 采用第一性原理密度泛函方法优化了 $\mathrm{LiMn}_{2} \mathrm{O}_{4}$ 尖晶石结构, 构建并计算了其低指数表面性质. 结果表明, 广义 梯度近似 $(\mathrm{GGA})$ 和自旋极化广义梯度近似 $(\mathrm{GGA}+\mathrm{U})$ 计算的 $\mathrm{LiMn}_{2} \mathrm{O}_{4}$ 晶体体相结构中, $\mathrm{Mn}$ 的 $\mathrm{d}$ 轨道选取有效 $\mathrm{U}$ 值时晶 格参数会变大. 但两种计算结果都没有显示出电荷有序和 Jahn-Teller 畸变的情况. $\mathrm{LiMn}_{2} \mathrm{O}_{4}$ 尖晶石结构缺 $\mathrm{Li}$ 条件下, (001)、(010)和(100)表面 $\mathrm{Li}$ 终端与其他终端相比表面能更低; (110)表面 $\mathrm{Mn} / \mathrm{O}$ 终端表面能较 $\mathrm{Li} / \mathrm{Mn} / \mathrm{O}$ 终端更低. 在所涉 及的低指数表面中(111)表面能最低, 表面重构后(111)表面能低至 $0.270 \mathrm{~J} / \mathrm{m}^{2}$, 是尖晶石结构中最稳定的切面. 关于反铁

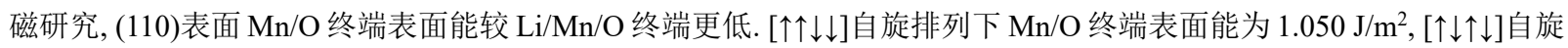
排列下 $\mathrm{Mn} / \mathrm{O}$ 终端表面能为 $1.061 \mathrm{~J} / \mathrm{m}^{2}$, 即(110)-反铁磁型表面在 $[\uparrow \uparrow \downarrow \downarrow]$ 自旋组态比 $[\uparrow \downarrow \uparrow \downarrow]$ 的磁性顺序下更加稳定. 通过 对(111)表面重构的研究，发现该表面欠配位的锰离子会与完全配位的锂离子通过位置交换，从而更加稳定. 重构表面 的平均锰氧化态降低, 会减少 Jahn-Teller 效应的产生. 除(111)表面外, 其它低指数表面在铁磁和反铁磁下的表面能相 似. 其中, (001)T3, (100)T1, (110)T1 和(111)T2 的表面结构在各自不同表面终端中具有最小的表面能. 本研究为理解 $\mathrm{LiMn}_{2} \mathrm{O}_{4}$ 材料容量衰减问题和实验提供理论计算参考, 有助于推动高性能锂电池材料的研究.

关键词 $\mathrm{LiMn}_{2} \mathrm{O}_{4}$ 尖晶石氧化物; 第一性原理计算; Jahn-Teller 效应; 低指数表面; 表面重构
\end{abstract}

\section{First-principles Study on Low Index Surface Structure Optimization and Surface Energy of $\mathrm{LiMn}_{2} \mathrm{O}_{4}$ Spinel Oxides}

\author{
Yuan Lu Jifen Wang* Huaqing Xie \\ (School of Sciences, Shanghai Polytechnic University; Shanghai Engineering Research Center of \\ Advanced Thermal Functional Materials, Shanghai 201209, China)
}

\begin{abstract}
The first principles density functional calculation method was used to calculate the index of low surface properties of $\mathrm{LiMn}_{2} \mathrm{O}_{4}$ spinel. Comparing with the bulk phase structures of $\mathrm{LiMn}_{2} \mathrm{O}_{4}$ crystals calculated by generalized gradient approximation (GGA) and generalized gradient approximation + on-site coulombic $(\mathrm{GGA}+\mathrm{U})$, it is found that the lattice parameters are obviously larger when the effective $U$ value for the d orbital of Mn. However, the structural parameters of charge order and Jahn-Teller distortion are not found in own calculation. The results show that the Li terminal surface energy is lower when the (001), (010) and (100) surfaces of $\mathrm{LiMn}_{2} \mathrm{O}_{4}$ spinel in the state of lacking Li. The surface energy of (110) $\mathrm{Mn} / \mathrm{O}$ terminal is lower than that of $\mathrm{Li} / \mathrm{Mn} / \mathrm{O}$ terminal in the state of lacking Li. The surface energy of (111) is the lowest in the low index surfaces. The surface energy of (111) after surface reconstruction is as low as $0.270 \mathrm{~J} / \mathrm{m}^{2}$, which is the most stable section of spinel structure in this work. Considering antiferromagnetism, the surface energies of $\mathrm{Mn} / \mathrm{O}$ terminal on

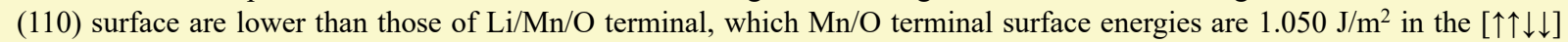
magnetic order and $1.061 \mathrm{~J} / \mathrm{m}^{2}$ in $[\uparrow \downarrow \uparrow \downarrow]$ magnetic order, respectively. The antiferromagnetic (110) surface is more stable in the spin configuration than in the magnetic order. We found that the structure of undercoordinated manganese ions on the surface are more stable by position exchange with the fully coordinated lithium ions by the observation of the surface reconstruction of (111). The Jahn-Teller effect is reduced when the average manganese oxidation state of the reconstructed surface is reduced. Except for (111) surface, the surface energies of other surfaces in the ferromagnetic state are similar to those in the antiferromagnetic state. Among them, the surface structures of (001)T3, (100)T1, (110)T1 and (111)T2 have the least surface energy among their own different surface terminals. This study provides theoretical calculation reference for understanding the capacity attenuation problem of $\mathrm{LiMn}_{2} \mathrm{O}_{4}$ materials and related experiments. It is also helpful to promote the research of high-performance lithium battery materials.
\end{abstract}

Keywords $\mathrm{LiMn}_{2} \mathrm{O}_{4}$ spinel oxide; first principle; Jahn-Teller effect; low exponent surface; surface reconstruction

* E-mail: wangjifen@sspu.edu.cn
Received May 14, 2021; published June 1, 2021.

Project supported by the National Natural Science Foundation of China (No. 51776116) and the Major Project of National Natural Science Foundation of China (No. 51590902).

项目受国家自然科学基金(No. 51776116)和国家自然科学基金重大项目(No. 51590902)资助. 


\section{1 引言}

随着科学的进展和社会的进步, 锂离子电池由于能 量密度高被认为是最有发展前景的电池材料之一. 自锂 离子电池投放市场以后, 被广泛运用于水力、火力、风 力和太阳能电站等储能电源系统, 目前已逐步向电动自 行车、新能源电动汽车等领域拓展. 其中正极材料 $\mathrm{LiMn}_{2} \mathrm{O}_{4}$ 因为安全性能良好、价格成本低廉和对环境友 好的优点, 有望取代 $\mathrm{LiCoO}_{2}$ 电池成为新一代高性能锂 电池材料 ${ }^{[1-4]}$. 然而 $\mathrm{LiMn}_{2} \mathrm{O}_{4}$ 存在的缺点也是不容忽视 的, 在高温情况下容量易衰减成为它广泛应用的制 约 ${ }^{[5-7]}$.

$\mathrm{LiMn}_{2} \mathrm{O}_{4}$ 存在电池容量衰减问题的主要原因是深度 充放电过程中材料晶格不稳定会发生畸变. 具体的电池 容量衰减原因包括 $\mathrm{Mn}$ 的溶解、Jahn-Teller 效应、氧的 缺陷和电解液的分解等. Mn 的溶解主要发生在充电后 期, 它会增加正极材料与导电剂之间的电阻 ${ }^{[8]}$. 电解液 中残留的水会与电解液盐发生反应, 导致锰离子的减 少 ${ }^{[9]}$. Jahn-Teller 效应主要发生在金属的化学反应中, 特 别是具有 $\mathrm{d}$ 轨道的金属配合物中. 在 $\mathrm{LiMn}_{2} \mathrm{O}_{4}$ 材料的深 充放电过程中, $\mathrm{Mn}^{3^{+}}$会产生 Jahn-teller 效应, 这被认为 是材料容量衰减最重要的原因 ${ }^{[10]}$. 关于 $\mathrm{LiMn}_{2} \mathrm{O}_{4}$ 材料容 量衰减问题有几个方面, 但是 Mn 的溶解和 Jahn-Teller 效应是关键因素. 本工作主要研究分析 $\mathrm{LiMn}_{2} \mathrm{O}_{4}$ 尖晶石 氧化物的低指数表面结构稳定性条件, 来减少 Jahn-Teller 效应的产生. 另外, 该材料因为 $\mathrm{Mn}^{3+}$ 原因产 生畸变, 结构将从原来的高对称性立方晶系转变为低对 称性立方晶系, 进而降低其材料的电容量.

$\mathrm{LiMn}_{2} \mathrm{O}_{4}$ 材料体系表面结构和表面能量的研究引起 了学界的重视, 以提高相关材料的稳定性. Benedek 和 Thackeray 在自旋极化广义梯度近似 $(\mathrm{GGA}+\mathrm{U})$ 近似下 对电极材料 $\mathrm{LiMn}_{2} \mathrm{O}_{4}$ 的低指数表面结构进行了模拟 ${ }^{[11]}$. 表面计算时预测了 $\mathrm{Mn}$ 端部(111)表面的重构, 构建出具 有平面协调的 $\mathrm{Mn}$ 端和 $\mathrm{Li} / \mathrm{Mn} / \mathrm{O}$ 端层来减少表面的净偶 极子. 考虑到所有低指数表面能计算, 最终发现以锂为 终止端的(001)表面能量最低. Karim 等 ${ }^{[12]}$ 通过计算优化 后磁性和电子状态下低指数(100), (110)和(111)面能量 来确定稳定下的 $\mathrm{LiMn}_{2} \mathrm{O}_{4}$ 材料表面. 采用第一性原理计 算过程中设置了相同的电子参数, 与其他表面切面相 比，(111)面这种选择性表面重构可以降低表面能量, 并 使该表面成为尖晶石结构中最稳定的切面. 最后, 基于 Wulff 构造利用表面总能量的最小化, 成功预测了 $\mathrm{LiMn}_{2} \mathrm{O}_{4}$ 材料粒子的平衡形状. 对 $\mathrm{LiMn}_{2} \mathrm{O}_{4}$ 材料理论研 究多在于计算晶体表面性质, 当它的表面吸附或是包覆 其他氧化物构成结合面时也应该会对材料的性能产生 影响. Ouyang 等 ${ }^{[13]}$ 采用从头算的方法计算了纯的和 $\mathrm{Al}_{2} \mathrm{O}_{3}$ 包覆下的 $\mathrm{LiMn}_{2} \mathrm{O}_{4}$ 材料(001)表面结构. 计算结果 表明 $\mathrm{Al}_{2} \mathrm{O}_{3}$ 包覆可以有效抑制 $\mathrm{LiMn}_{2} \mathrm{O}_{4}$ 材料表面 $\mathrm{Mn}^{3+}$ 的形成, 致使 $\mathrm{LiMn}_{2} \mathrm{O}_{4}$ 材料电循环性能提升. 但是
$\mathrm{Al}_{2} \mathrm{O}_{3}$ 包覆也存在缺陷, 它会降低电极表面 $\mathrm{Li}$ 的通过率. 为了解决这个问题，他们发现可以用羟基和有机分子共 同包覆来完善不足. 这就需要研究者通过实验和理论计 算相结合，找到极具潜力的氧化物和羟基做进一步的研 究工作. 王婷等 ${ }^{[14]}$ 使用 $\mathrm{X}$ 射线衍射/扫描电子显微镜 (XRD/SEM) 技术研究了固相反应合成的 $\mathrm{LiMn}_{2} \mathrm{O}_{4}$ 表面 结构和形貌特征, 并通过电化学测试研究 $\mathrm{LaF}_{3}$ 修饰下 $\mathrm{LiMn}_{2} \mathrm{O}_{4}$ 在不同温度下的电化学性能. $\mathrm{LaF}_{3}$ 修饰下 $\mathrm{LiMn}_{2} \mathrm{O}_{4}$ 结构不变, 电化学性能和循环可逆性得到提升. $\mathrm{Bi}$ 等 ${ }^{[15]}$ 在 $\mathrm{LiMn}_{2} \mathrm{O}_{4}$ 阴极表面上涂 LLZNO 电解质后发现 增强了电化学性能, 而且有助于抑制 $\mathrm{Mn}$ 的溶解.

为了研究 $\mathrm{LiMn}_{2} \mathrm{O}_{4}$ 尖晶石氧化物的低指数表面结 构稳定性, 本工作计算了 $\mathrm{LiMn}_{2} \mathrm{O}_{4}$ 尖晶石氧化物的表面 能. 对比了本体和表面在铁磁(FM)和反铁磁(AFM)时的 情况, 研究了磁性顺序和 U 值对 $\mathrm{LiMn}_{2} \mathrm{O}_{4}$ 表面能大小的 影响. 计算分析出 $\mathrm{LiMn}_{2} \mathrm{O}_{4}$ 尖晶石氧化物的低指数表面 结构稳定性条件, 确定它在哪种表面情况下最具有热力 学稳定性, 找到在现实电化学条件下可能存在的结构研 究新起点.

\section{2 计算方法}

\section{1 电子结构}

采用从头计算软件包 VASP 对 $\mathrm{LiMn}_{2} \mathrm{O}_{4}$ 尖晶石氧化 物进行自旋极化密度泛函理论和 Hubbard U(DFT+U) 的超软赝势平面波方法优化其结构并计算相关低指数 表面性质 ${ }^{[16-17]}$. 在本研究中, 选取空间群结构 $F d-3 m$ 的 $\mathrm{LiMn}_{2} \mathrm{O}_{4}$ 晶胞模型, 如图 1 所示 ${ }^{[13]}$. 优化前结构模型 $\mathrm{LiMn}_{2} \mathrm{O}_{4}$ 体相为 $a=b=c=0.8238 \mathrm{~nm}$ 的立方对称 $(\alpha=\beta$ $=\gamma=90^{\circ}$ ) 晶格常数. 利用晶格常数和优化后的结构计 算 $\mathrm{LiMn}_{2} \mathrm{O}_{4}$ 尖晶石不同低指数表面的表面能, 研究它们 的结构和稳定性. 计算采用密度泛函理论(DFT)的自旋 极化广义梯度近似 $(\mathrm{GGA}+\mathrm{U})$ 进行，并采用 $550 \mathrm{eV}$ 的截 断能作为平面波基础设置. 在自旋不变的 $\mathrm{GGA}+\mathrm{U}$ 方 法中, 截断能量为 $550 \mathrm{eV}, \mathrm{Mn}$ 的 $\mathrm{d}$ 轨道选取有效 $\mathrm{U}$ 值 5 $\mathrm{eV}^{[12]}$. 由于局部密度近似(LDA)或广义梯度近似(GGA) 的 DFT 计算中锂离子电池材料会被大大低估带隙, 选 用 GGA+U 方法可以减少带隙误差的产生, 让理论计 算更加接近实验值 ${ }^{[18]}$. 关于 $U$ 值选取发现在 $3.5 \sim 5.5$ $\mathrm{eV}$ 之间对于计算结果相近影响不大, 所以本工作设置 时选取 $5 \mathrm{eV}$ 对后续的表面进行计算. 计算 $\mathrm{k}$ 点网格采用 $3 \times 3 \times 3$ 的 $\mathrm{LiMn}_{2} \mathrm{O}_{4}$ 体相和 $3 \times 3 \times 1$ 的 $\mathrm{LiMn}_{2} \mathrm{O}_{4}$ 表面平 板晶胞进行表面计算. 表面计算中采用高斯展宽, 展宽 宽度为 $0.1 \mathrm{eV}$. 平板计算中的所有原子进行完全的离子 弛豫，原子间相互作用力的收玫标准设置为 $0.2 \mathrm{eV} / \mathrm{nm}$, 原子能量收敛于 $1.0 \times 10^{-5} \mathrm{eV}$. 在所有计算中，使用收 敛标准测试并考虑了锰原子的铁磁(FM) 和反铁磁序 (AFM)对表面能的影响. 


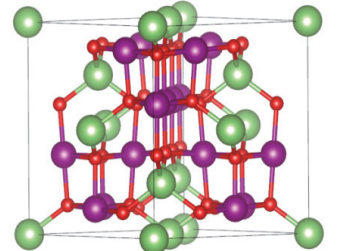

图 $1 \mathrm{LiMn}_{2} \mathrm{O}_{4}$ 立方尖晶石晶胞结构, 指示 $\mathrm{Li}($ 绿色 $) 、 \mathrm{Mn}$ (紫红)和 $\mathrm{O}($ 红 色)原子的位置

Figure1 $\mathrm{LiMn}_{2} \mathrm{O}_{4}$ cubic spinel structure, indicating the positions of atoms $\mathrm{Li}$ (green), $\mathrm{Mn}$ (magenta), and $\mathrm{O}$ (red)

\section{2 热力学稳定相图计算}

在计算相图和表面稳定性时, 采用 CALPHAD 方 法, 计算平衡相图 ${ }^{[19]}$. 对于非化学计量的板层结构表面 能计算时, 参考了开放量子材料数据库 (OQMD) 中 Li-Mn-O 化学空间中除 $\mathrm{LiMn}_{2} \mathrm{O}_{4}$ 外, 基态下大块体系 $\mathrm{Mn} 、 \mathrm{Li}_{2} \mathrm{O} 、 \mathrm{Li}_{2} \mathrm{O}_{2} 、 \mathrm{MnO}_{2} 、 \mathrm{MnO}_{4} 、 \mathrm{Mn}_{2} \mathrm{O}_{3} 、 \mathrm{LiMnO}_{2}$ 和 $\mathrm{Li}_{2} \mathrm{MnO}_{3}$ 的最稳定形式 ${ }^{[20-21]}$. 根据锂锰氧化物在 DFT 基 态时计算出的 $\Delta G_{\mathrm{f}}$ 值构建 Li-Mn-O 空间的三元相图. 图 2 是对于 $T=0 \mathrm{~K}$ 时的封闭体系, 通过求解对应相区的每 一个稳定相得到 $\mathrm{O}$ 角附近的 Li-Mn-O 基态相图. 因此, 在相图上从 $\mathrm{Li}-\mathrm{Mn}-\mathrm{O}$ 画出的所有线都与化合物相联系 并处于平衡状态. 在体相 $\mathrm{LiMn}_{2} \mathrm{O}_{4}$ 的稳定相区会受其与 下列物质的平衡限制: $\mathrm{MnO}_{2} 、 \mathrm{Mn}_{2} \mathrm{O}_{3} 、 \mathrm{Mn}_{2} \mathrm{O}_{4} 、 \mathrm{LiMnO}_{2}$ 、 $\mathrm{Li}_{2} \mathrm{MnO}_{3} 、 \mathrm{LiMnO}_{2} 、 \mathrm{O}$. 如图 2 所示, 稳定化合物(点)、 两相混合物(连线)、三相平衡(三角形). 参照 $\mathrm{Li}-\mathrm{Mn}-\mathrm{O}$ 的 三元相图, 非化学计量的组分和参考相混合物如表 1 所 示.

表 1 由三相图整理的非化学计量平板组分情况

Table 1 Non-stoichiometric plate composition as summarized by three-phase diagram

\begin{tabular}{|c|c|c|}
\hline 化学式 & 平板混合物组分 & $\begin{array}{l}\text { 锂镇氧化物 } \\
\text { 基态能量 } \Delta G_{\mathrm{f}}\end{array}$ \\
\hline \multicolumn{3}{|c|}{ Slab: $\mathbf{L i}_{1+x} \mathbf{M n}_{2} \mathbf{O}_{4}$} \\
\hline $\mathrm{Li}_{10} \mathrm{Mn}_{16} \mathrm{O}_{32}$ & $\begin{array}{c}\text { 4.67 } \mathrm{LiMn}_{2} \mathrm{O}_{4}+2.67 \mathrm{Li}_{2} \mathrm{MnO}_{3} \\
+1.33 \mathrm{Mn}_{3} \mathrm{O}_{4}\end{array}$ & $\begin{array}{l}E_{\mathrm{LiMn}_{2} \mathrm{O}_{4}}= \\
-47.17 \mathrm{eV}\end{array}$ \\
\hline $\mathrm{Li}_{18} \mathrm{Mn}_{32} \mathrm{O}_{64}$ & $\begin{array}{c}12.67 \mathrm{LiMn}_{2} \mathrm{O}_{4}+ \\
2.67 \mathrm{Li}_{2} \mathrm{MnO}_{3}+1.33 \mathrm{Mn}_{3} \mathrm{O}_{4}\end{array}$ & $\begin{array}{l}E_{\mathrm{Li}_{2} \mathrm{MnO}_{3}}= \\
-36.65 \mathrm{eV}\end{array}$ \\
\hline $\mathrm{Li}_{26} \mathrm{Mn}_{48} \mathrm{O}_{96}$ & $\begin{array}{c}20.67 \mathrm{LiMn}_{2} \mathrm{O}_{4}+ \\
2.67 \mathrm{Li}_{2} \mathrm{MnO}_{3}+1.33 \mathrm{Mn}_{3} \mathrm{O}_{4}\end{array}$ & $E_{\mathrm{Mn}_{3} \mathrm{O}_{4}}=-52.30 \mathrm{eV}$ \\
\hline \multicolumn{3}{|c|}{ Slab: $\mathrm{LiMn}_{2+x} \mathrm{O}_{4}$} \\
\hline $\mathrm{Li}_{8} \mathrm{Mn}_{20} \mathrm{O}_{32}$ & $8 \mathrm{LiMnO}_{2}+4 \mathrm{Mn}_{3} \mathrm{O}_{4}$ & $\begin{array}{l}E_{\mathrm{Li}_{10 \mathrm{Mn}} \mathrm{M}_{32}}= \\
-387.71 \mathrm{eV}\end{array}$ \\
\hline $\mathrm{Li}_{16} \mathrm{Mn}_{36} \mathrm{O}_{64}$ & $\begin{array}{c}5.33 \mathrm{LiMn}_{2} \mathrm{O}_{4}+5.33 \mathrm{Li}_{2} \mathrm{MnO}_{3} \\
+6.67 \mathrm{Mn}_{3} \mathrm{O}_{4}\end{array}$ & $\begin{array}{l}E_{\mathrm{Li}_{16} \mathrm{Mn}_{36} \mathrm{O}_{64}}= \\
-795.80 \mathrm{eV}\end{array}$ \\
\hline $\mathrm{Li}_{24} \mathrm{Mn}_{52} \mathrm{O}_{96}$ & $\begin{array}{c}13.33 \mathrm{LiMn}_{2} \mathrm{O}_{4}+ \\
5.33 \mathrm{Li}_{2} \mathrm{MnO}_{3}+6.67 \mathrm{Mn}_{3} \mathrm{O}_{4}\end{array}$ & $\begin{array}{l}E_{\mathrm{Li}_{24 \mathrm{Mn}} 2 \mathrm{O} 96}= \\
-1172.98 \mathrm{eV}\end{array}$ \\
\hline
\end{tabular}

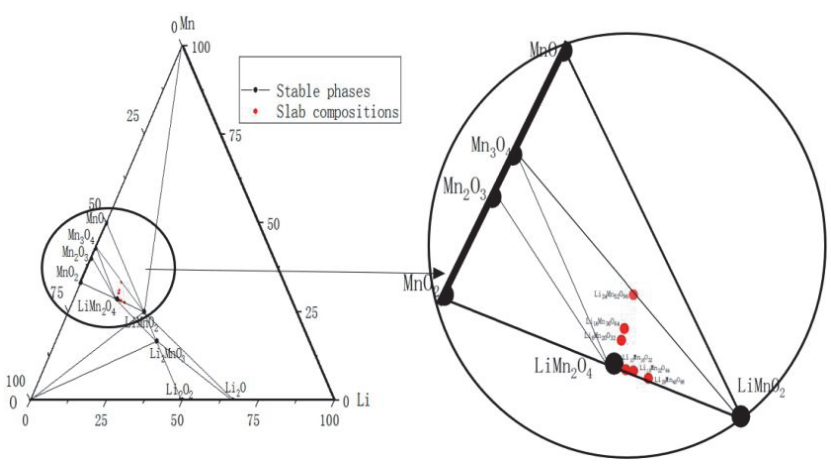

图 2 三元相图表明稳定的化合物结合体 Li-Mn-O

Figure 2 Ternary phase diagram, indicating stable compound complex Li-Mn-O

\section{3 表面能计算}

一般化学计量情况下，用 DFT 来计算表面能的方 法是从含有相同原子数量 $\mathrm{LiMn}_{2} \mathrm{O}_{4}$ 的平板的能量中减 去相同数量的体相 $\mathrm{LiMn}_{2} \mathrm{O}_{4}$ 的能量, 再除以包括上下两 面的平板表面积. 因此, 表面能计算公式如下 ${ }^{[22]}$ :

$$
E_{\text {surf }}=\frac{E_{\text {slab }}-N E_{\text {bulk }}}{2 A}
$$

其中, $E_{\mathrm{slab}}$ 为平板结构优化后的能量; $E_{\mathrm{bulk}}$ 为体相结构优 化后的能量; $N$ 为表面平板中的原子数; $A$ 为平板的单基 底面积.

非化学计量比的表面能计算, 在体相能量计算时要 参考 $\mathrm{Li}-\mathrm{Mn}-\mathrm{O}(T=0 \mathrm{~K}$ 时)基态相图. 实际上, 有时候 Li-Mn-O 粒子的合成条件可能偏离封闭的热力学稳定体 系而与外界氧气或锂源产生反应. 因此, 在三元相图中, 化学势大小可以依照不同的合成条件在外部进行控制.

计算 $\mathrm{LiMn}_{2} \mathrm{O}_{4}$ 尖晶石(100), (010), (001), (110)和 (111)表面的表面能. 在进行表面弛豫计算中采用了 Tasker 准则 ${ }^{[23]}$. (001)表面真空厚度设为 $1.3 \mathrm{~nm}$, 选择 8 层厚度的平板计算. (111)面的真空层设置为 $2.4 \mathrm{~nm}$, 避 免上下层原子间产生作用. 对于已重构的(111)表面中 间部分原子交换位置. 在不同低指数表面, 研究 $\mathrm{Li}$, $\mathrm{Mn} / \mathrm{O}$ 以及 $\mathrm{Li} / \mathrm{Mn} / \mathrm{O}$ 终端在不同平板厚度下的表面情况.

\section{3 结果与讨论}

\section{1 $\mathrm{LiMn}_{2} \mathrm{O}_{4}$ 体相}

$\mathrm{LiMn}_{2} \mathrm{O}_{4}$ 体相室温下呈现尖晶石结构. 表 2 是 $\mathrm{LiMn}_{2} \mathrm{O}_{4}$ 尖晶石的晶格参数值. GGA 计算所得晶格 $(a=$ $b=c=0.8146 \mathrm{~nm})$ 略低于实验值 $(a=b=c=0.8238 \mathrm{~nm})$. 而 $\mathrm{DFT}+\mathrm{U}$ 计算所得晶格参数 $(a=b=c=0.8419 \mathrm{~nm})$ 较 GGA 计算结果增大了 $0.0273 \mathrm{~nm}$, 从而高于实验测量值 $2 \%$. 这两种情况下都没有显示出表明电荷有序和 Jahn-Teller 畸变的结构参数. 合理的 $\mathrm{AF}$ 有序和电子定 位(GGA+U)会导致产生 Jahn-Teller 畸变 ${ }^{[24-26] . ~}$ 
表 $2 \mathrm{LiMn}_{2} \mathrm{O}_{4}$ 尖晶石的晶格参数 $a 、 b$ 和 $c$

Table 2 Experimental and computational lattice parameters $a, b$ and $c$ values of $\mathrm{LiMn}_{2} \mathrm{O}_{4}$ spinel

\begin{tabular}{cccccc}
\hline $\mathrm{LiMn}_{2} \mathrm{O}_{4}$ & 实验值 $(\mathrm{nm})$ & $\begin{array}{c}\mathrm{GGA} \\
(\mathrm{nm})\end{array}$ & $\begin{array}{c}\mathrm{GGA}+\mathrm{U} \\
(\mathrm{nm})\end{array}$ & $\begin{array}{c}\mathrm{GGA} \\
(\mathrm{nm})\end{array}$ & $\begin{array}{c}\mathrm{GGA}+\mathrm{U} \\
(\mathrm{nm})\end{array}$ \\
\hline$a$ & $0.8238^{[27]}$ & 0.8146 & 0.8419 & $0.809^{[12]}$ & $0.843^{[12]}$ \\
$b$ & $0.8238^{[27]}$ & 0.8146 & 0.8419 & $0.809^{[12]}$ & $0.843^{[12]}$ \\
$c$ & $0.8238^{[27]}$ & 0.8146 & 0.8419 & $0.809^{[12]}$ & $0.843^{[12]}$ \\
\hline
\end{tabular}

\section{2 表面结构}

\subsection{1 (001)表面和(010)表面}

$\mathrm{Li}$ 化物结构优化后会导致 $\mathrm{MnO}$ 层出现较弱的 $\mathrm{Mn}$ 原子链聚合, 所以要对不同的表面终端进行计算分析. 对(001)表面采用三种不同的方法来构造切面. 图 3 为 (001)表面和(010)表面原子结构和表面能量结果图. 图 3 (a)在板的两侧有不对称表面的化学计量板(T1), 其中顶 面由锂原子组成, 而底层表面暴露镇氧层. (b)具有对称 等效表面的化学计量板(T2), 上下基底面都是 $\mathrm{Li}$ 终端. 上下两个表面因为都是 $\mathrm{Li}$ 层, 相对于 $\mathrm{T} 1$ 的简单解理终 端是欠协调的. (c)在每一侧有相同末端的非化学计量板 (T3)，上下基底面都是 $\mathrm{Li}$ 终端. 其中 $\mathrm{Li}$-和 $\mathrm{Mn} / \mathrm{O}$-终止端 的 $\mathrm{LiMn}_{2} \mathrm{O}_{4}(001)$ 表面统一设置了 $1.3 \mathrm{~nm}$ 的真空厚度. (a)

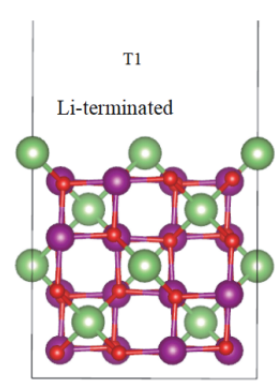

$\mathrm{Mn} / \mathrm{O}$-terminated (b)

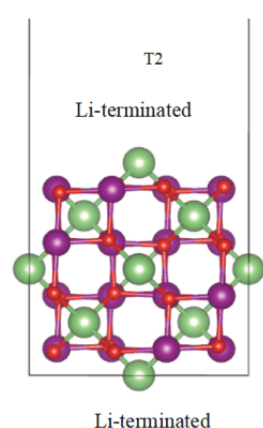

(c)

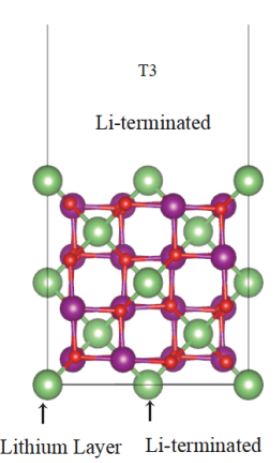

图 3 (001)和(010)表面结构图, 尖晶石 $\mathrm{LiMn}_{2} \mathrm{O}_{4}(001)$ 表面的原子结 构: $\mathrm{T} 1$ : 非对称 $\mathrm{Li}$-和 $\mathrm{Mn} / \mathrm{O}$-终端的 $\mathrm{Li}_{8} \mathrm{Mn}_{16} \mathrm{O}_{32}$ 表面 (八层); $\mathrm{T} 2$ : 对称 $\mathrm{Li}$ 终端的 $\mathrm{Li}_{8} \mathrm{Mn}_{16} \mathrm{O}_{32}$ 表面(九层); $\mathrm{T} 3$ : 对称 $\mathrm{Li}$ 终端, 非化学计量 $\mathrm{Li}_{10} \mathrm{Mn}_{16} \mathrm{O}_{32}$ 表面(九层). $\mathrm{Li}$ (绿色)、 $\mathrm{Mn}$ (紫红)和 $\mathrm{O}$ (红色)

Figure 3 (001) Surface and (010) surface structure, atomic structure of spinel $\mathrm{LiMn}_{2} \mathrm{O}_{4}(001)$ surface: T1: Asymmetric Li- and $\mathrm{Mn} / \mathrm{O}$-terminal $\mathrm{Li}_{8} \mathrm{Mn}_{16} \mathrm{O}_{32}$ surface (eight layers); $\mathrm{T} 2: \mathrm{Li}_{8} \mathrm{Mn}_{16} \mathrm{O}_{32}$ surface (nine layers) of symmetrical Li terminal; T3: symmetrical Li terminal, non-stoichiometric $\mathrm{Li}_{10} \mathrm{Mn}_{16} \mathrm{O}_{32}$ surface (nine layers). Li (green), Mn (magenta) and $\mathrm{O}$ (red)

\subsection{2 (100)表面}

表 3 是 $\mathrm{LiMn}_{2} \mathrm{O}_{4}(100)$ 的表面结构和顶部视角. 从表 面结构的顶部观察, 呈现出一个排列非常紧密的蜂窝 状. 从表面结构的顶部观察, $\mathrm{Mn} / \mathrm{O}$ 终端原子排列松散有 较多断键空间.
表 $3 \mathrm{LiMn}_{2} \mathrm{O}_{4}(100)$ 的表面结构和顶部视角

Table 3 Surface structure and top view of $\mathrm{LiMn}_{2} \mathrm{O}_{4}(100)$

\begin{tabular}{|c|c|c|}
\hline Terminated & Atomic structure & Top view \\
\hline \multirow[t]{2}{*}{ Li terminated } & 0 & \\
\hline & $\mathrm{T} 1$ & \\
\hline \multirow[t]{3}{*}{$\mathrm{Mn} / \mathrm{O}$ terminated } & & \\
\hline & :20.: & 0 \\
\hline & $\mathrm{T} 2$ & \\
\hline
\end{tabular}

\section{2 .3 (110)表面}

图 4 是 $\mathrm{LiMn}_{2} \mathrm{O}_{4}(110)$ 的表面结构, 主要研究了 $\mathrm{Mn} / \mathrm{O}$ 和 $\mathrm{Li} / \mathrm{Mn} / \mathrm{O}$ 终端两种情况. 该表面板层以 $\mathrm{Mn}_{x} \mathrm{O}_{2 x}$ 和 $\mathrm{Li}_{x} \mathrm{Mn}_{x} \mathrm{O}_{2 x}$ 的化学计量单元重复出现. (a)

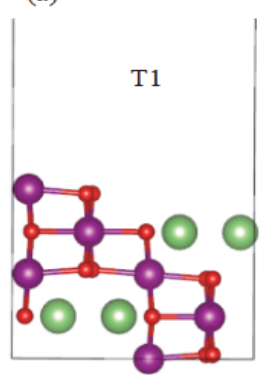

(b)

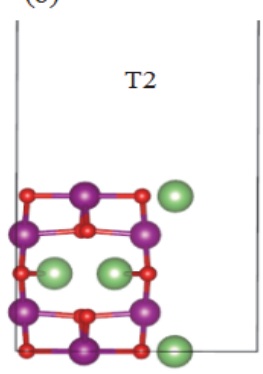

图 4 (110)表面原子结构图: (a) $\mathrm{Mn} / \mathrm{O}$ 终端的 $\mathrm{Li}_{8} \mathrm{Mn}_{16} \mathrm{O}_{32}$ 表面(五层); (b) $\mathrm{Li} / \mathrm{Mn} / \mathrm{O}$ 终端的 $\mathrm{Li}_{8} \mathrm{Mn}_{16} \mathrm{O}_{32}$ 表面(五层). $\mathrm{Li}$ (绿色)、Mn(紫红)和 $\mathrm{O}$ (红 色)

Figure 4 (110) Surface structure diagram: (a) $\mathrm{Mn} / \mathrm{O}$ terminal $\mathrm{Li}_{8} \mathrm{Mn}_{16} \mathrm{O}_{32}$ surface (five layers); (b) $\mathrm{Li} / \mathrm{Mn} / \mathrm{O}$ terminal $\mathrm{Li}_{8} \mathrm{Mn}_{16} \mathrm{O}_{32}$ surface (five layers). Li (green), Mn (magenta), and O (red)

\subsection{4 (111)表面}

图 5 给出了尖晶石 $\mathrm{LiMn}_{2} \mathrm{O}_{4}(111)$ 表面的原子结构 图. 其中图 5(a)是不对称的 $\mathrm{Li} / \mathrm{Mn} / \mathrm{O}$ 端和 $\mathrm{Li} / \mathrm{O}$ 端表面的 化学计量板( $\mathrm{T} 1)$; 图 5(b) 是具有对称等效 $\mathrm{Li} / \mathrm{Mn} / \mathrm{O}$ 端表 面的化学计量板(T2); 图 5(c)相较于 T2 的情况, 在底层 增加 4 个 $\mathrm{Mn}$ 原子形成 $\mathrm{Li} / \mathrm{Mn} / \mathrm{O}$ 终端的非化学计量板层 (T3). 晶体材料表面通过原子的重新排列进行重构, 这 样可以有效降低体系的表面能量 ${ }^{[28-32]}$. 图 5 可以看出优 化后的尖晶石 $\mathrm{LiMn}_{2} \mathrm{O}_{4}(111)$ 表面以化学计量重复单元 序列组成, 每一层都包含一种单一的、不同的化学物质. 这种结构特征同 Tasker III 型强极性氧化物表面一致, 是由纯的单层金属离子和纯的单层氧交替组成 ${ }^{[33-35]}$. $\mathrm{LiMn}_{2} \mathrm{O}_{4}(111)$ 表面的化学计量重复单元由以下分层序列 组成: $\mathrm{Li}_{x}, \mathrm{Mn}_{x}, \mathrm{O}_{4 x}, \mathrm{Mn}_{3 x}, \mathrm{O}_{4 x}, \mathrm{Li}_{x}$. 
(a)

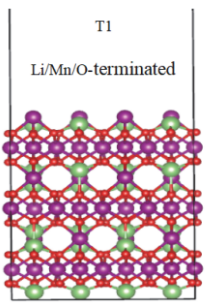

Li/o-terminated (b)

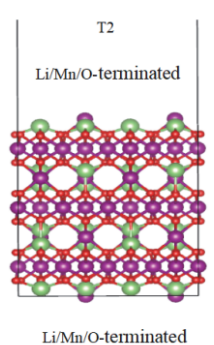

(c)

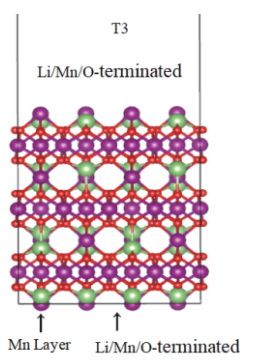

图 5 尖晶石 $\mathrm{LiMn}_{2} \mathrm{O}_{4}(111)$ 表面的原子结构: (a) 不对称 $\mathrm{Li} / \mathrm{Mn} / \mathrm{O}$ 和 $\mathrm{Li} / \mathrm{O}$ 端的 $\mathrm{Li}_{24} \mathrm{Mn}_{48} \mathrm{O}_{96}$ 表面; (b) 对称等效 $\mathrm{Li} / \mathrm{Mn} / \mathrm{O}$ 端的 $\mathrm{Li}_{24} \mathrm{Mn}_{48} \mathrm{O}_{96}$; (c) 在底层增加 4 个 $\mathrm{Mn}$ 原子的非化学计量板层 $\mathrm{Li}_{24} \mathrm{Mn}_{52} \mathrm{O}_{96}$. 表面 $\mathrm{Li}$ (绿 色)、 $\mathrm{Mn}$ (紫红)和 $\mathrm{O}$ (红色)

Figure 5 Atomic structure of spinel $\mathrm{LiMn}_{2} \mathrm{O}_{4}(111)$ surface: (a) asymmetric $\mathrm{Li} / \mathrm{Mn} / \mathrm{O}$ and $\mathrm{Li} / \mathrm{O}$ terminal $\mathrm{Li}_{24} \mathrm{Mn}_{48} \mathrm{O}_{96}$ surface; (b) $\mathrm{Li}_{24} \mathrm{Mn}_{48} \mathrm{O}_{96}$ with symmetrical equivalent $\mathrm{Li} / \mathrm{Mn} / \mathrm{O}$ terminal; (c) Non-stoichiometric laminae $\mathrm{Li}_{24} \mathrm{Mn}_{52} \mathrm{O}_{96}$ with $4 \mathrm{Mn}$ atoms added in the bottom layer. Surface $\mathrm{Li}$ (green), Mn (magenta) and $\mathrm{O}$ (red)

图 6 是重构的(111) $\mathrm{LiMn}_{2} \mathrm{O}_{4} \mathrm{~T} 1$ 表面的原子结构图, 交换间隔原子层的 $\mathrm{Li}$ 原子和 $\mathrm{Mn}$ 原子位置并逐步增加板 层厚度.
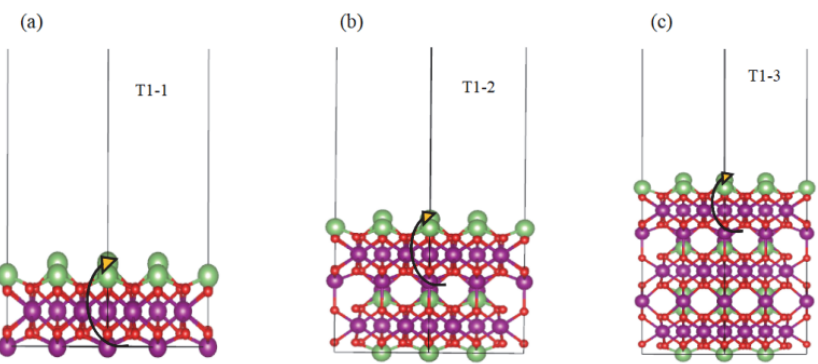

图 6 重构的(111) $\mathrm{LiMn}_{2} \mathrm{O}_{4} \mathrm{~T} 1$ 表面的原子结构图: (a) 顶面重构的 $\mathrm{Li}_{8} \mathrm{Mn}_{16} \mathrm{O}_{32}$ 表面(T1-1); (b) $\mathrm{Li}_{16} \mathrm{Mn}_{32} \mathrm{O}_{64}$ 表面(T1-2); (c) $\mathrm{Li}_{24} \mathrm{Mn}_{48} \mathrm{O}_{96}$ 表面 (T1-3)

Figure 6 Atomic structure diagram of reconstructed (111) surface of $\mathrm{LiMn}_{2} \mathrm{O}_{4} \mathrm{~T} 1$ : (a) surface of $\mathrm{Li}_{8} \mathrm{Mn}_{16} \mathrm{O}_{32}$ reconstructed at the top surface (T1-1); (b) $\mathrm{Li}_{16} \mathrm{Mn}_{32} \mathrm{O}_{64}$ surface (T1-2); (c) $\mathrm{Li}_{24} \mathrm{Mn}_{48} \mathrm{O}_{96}$ surface (T1-3)

图 7 是上下表面重构的(111) $\mathrm{LiMn}_{2} \mathrm{O}_{4} \mathrm{~T} 2$ 和 $\mathrm{T} 3$ 原子 结构. 图 7(a)和 7(b)分别是对 T2 情形下上下表面的重 构, 主要通过终端的四面体锂原子去交换八面体表面位 置中的锰原子来重建(111)表面. 图 7(c)和 7(d)是对 T3 情形的重构.

\section{3 各表面能对比(FM 和 AFM)}

表 4 是 $\mathrm{LiMn}_{2} \mathrm{O}_{4}$ 各低指数表面在铁磁和反铁磁下的 表面能量表. 表 4 列出了本工作研究的所有表面的表面 能, 并与文献的计算值进行了比较. 表面能在不同的研 究中结果值明显不同, 这可能是由于不同的结构和磁性 参数, 如固定中间原子层数或锰的铁磁、反铁磁排列. 除了(111)取向的表面结构外, 其它低指数表面在铁磁 和反铁磁下的表面能相似. 对于铁磁和反铁磁结构, (001)T3, (100)T1, (110)T1 和(111)T2 的表面结构在几种 不同的表面终端中具有最小的表面能.

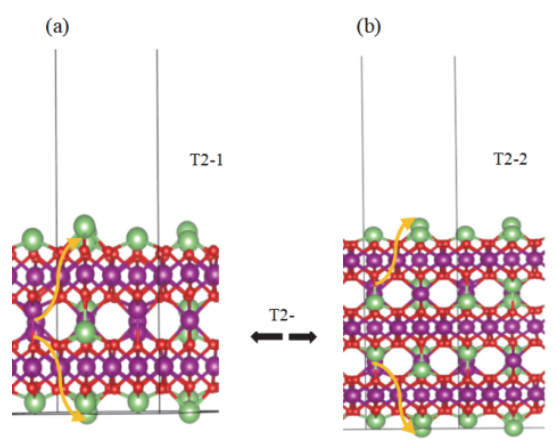

(c)

(d)

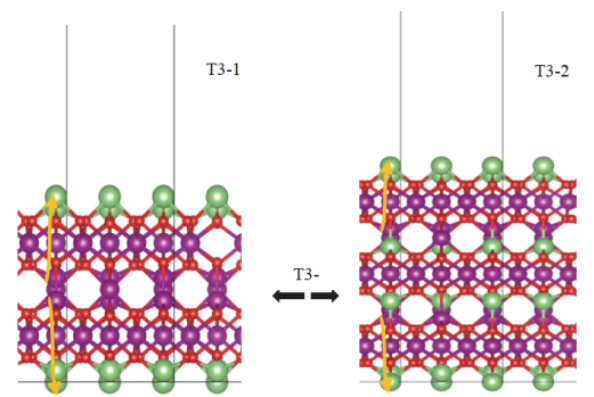

图 7 上下表面重构的(111) $\mathrm{LiMn}_{2} \mathrm{O}_{4} \mathrm{~T} 2$ 和 $\mathrm{T} 3$ 表面的原子结构图: (a) $\mathrm{Li}_{16} \mathrm{Mn}_{32} \mathrm{O}_{64}$ 表面(T2-1); (b) $\mathrm{Li}_{24} \mathrm{Mn}_{48} \mathrm{O}_{96}$ 表面(T2-2); (c) $\mathrm{Li}_{16} \mathrm{Mn}_{36} \mathrm{O}_{64}$ 表 面(T3-1). (d) $\mathrm{Li}_{24} \mathrm{Mn}_{52} \mathrm{O}_{96}$ 表面(T3-2)

Figure 7 Atomic structure diagram of (111) $\mathrm{LiMn}_{2} \mathrm{O}_{4} \mathrm{~T} 2$ and T3 surfaces reconstructed from upper and lower surfaces: (a) $\mathrm{Li}_{16} \mathrm{Mn}_{32} \mathrm{O}_{64}$ surface (T2-1); (b) $\mathrm{Li}_{24} \mathrm{Mn}_{48} \mathrm{O}_{96}$ surface (T2-2); (c) $\mathrm{Li}_{16} \mathrm{Mn}_{36} \mathrm{O}_{64}$ surface (T3-1); (d) $\mathrm{Li}_{24} \mathrm{Mn}_{52} \mathrm{O}_{96}$ Surface (T3-2)

表 4 使用 $\mathrm{GGA}+\mathrm{U}$ 计算(100), (010), (001), (110)和(111)低指数方向 的 $\mathrm{LiMn}_{2} \mathrm{O}_{4}$ 表面能(单位: $\mathrm{J} / \mathrm{m}^{2}$ )

Table 4 The surface energies of $\mathrm{LiMn}_{2} \mathrm{O}_{4}$ in the low index directions of (100), (010), (001), (110) and (111) are calculated using GGA+U (unit: $\left.\mathrm{J} / \mathrm{m}^{2}\right)$

\begin{tabular}{lcccc}
\hline Surface structure & $\begin{array}{c}\text { Surface } \\
\text { energy (FM) }\end{array}$ & $\begin{array}{c}\text { Surface energy } \\
(\mathrm{AFM})\end{array}$ & $\begin{array}{c}\text { Literature } \\
\text { reference }\end{array}$ \\
\hline$(001)$ & $\mathrm{T} 1$ & 0.598 & 0.628 & $0.860^{[36]}$ \\
& $\mathrm{T} 2$ & 0.600 & 0.626 & $0.840^{[36]}$ \\
& $\mathrm{T} 3$ & 0.597 & 0.615 & $0.720^{[36]}$ \\
$(100)$ & $\mathrm{T} 1$ & 0.606 & 0.654 & $0.870^{[12]}$ \\
& $\mathrm{T} 2$ & 1.127 & 1.195 & $1.280^{[12]}$ \\
$(110)$ & $\mathrm{T} 1$ & 1.026 & 1.050 & $1.410^{[12]}$ \\
& $\mathrm{T} 2$ & 1.971 & 1.969 & $1.760^{[12]}$ \\
$(010)$ & $\mathrm{T} 1$ & 0.599 & 0.668 & $1.300^{[12]}$ \\
& $\mathrm{T} 2$ & 0.603 & 0.667 & $0.960^{[12]}$ \\
& $\mathrm{T} 3$ & 0.651 & 0.644 & $\mathrm{~N} / \mathrm{A}$ \\
$(111)$ & $\mathrm{T} 1$ & 1.005 & 1.299 & $1.290^{[11]}$ \\
& $\mathrm{T} 2$ & 0.812 & 0.896 & $0.850^{[11]}$ \\
& $\mathrm{T} 3$ & 1.458 & 1.588 & $\mathrm{~N} / \mathrm{A}$ \\
\hline
\end{tabular}

\subsection{1 (001)和(010)表面能}

图 8 是非对称 $\mathrm{Li}$-和 $\mathrm{Mn} / \mathrm{O}$-终端的 $\mathrm{Li}_{8} \mathrm{Mn}_{16} \mathrm{O}_{32}(001)$ 和(010)表面. 两个表面情况都是 T1 表面能和 T2 相近, 而非化学计量 $\mathrm{T} 3 \mathrm{Li}_{10} \mathrm{Mn}_{16} \mathrm{O}_{32}$ 表面能量最低. (001)、(010) 两个表面能量比较, T(001)表面更稳定. 存在能量差异 的原因是 $\mathrm{T}(001)$ 表面 $\mathrm{Mn}$ 原子和 $\mathrm{O}$ 原子相邻, 表面的 Mn 原子会呈现 +3 价. 富锂层状氧化物材料如果缺乏 
稳定的锂表面结构会抑制表面副反应和层状到尖晶石 的转变, 是容量衰退的关键问题 ${ }^{[37-38]}$. 经计算发现(001) 表面 $\mathrm{Li}$ 终端的表面能更低, 说明在底层缺锂条件下的 $\mathrm{Mn} / \mathrm{O}$ 终端更加稳定. 添加额外锂离子后的非化学计量 比 T3-(001)表面能会降低, 这是因为该表面是三相图中 化学势最低的, 因此最稳定.

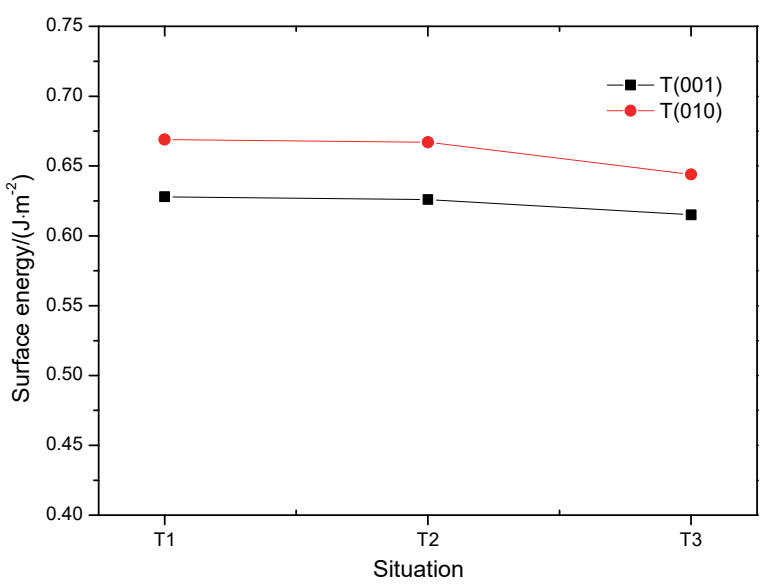

图 $8 \mathrm{LiMn}_{2} \mathrm{O}_{4}(001)$ 和 $\mathrm{LiMn}_{2} \mathrm{O}_{4}(010)$ 的表面能图

Figure 8 Surface energy diagrams of $\mathrm{LiMn}_{2} \mathrm{O}_{4}(001)$ and $\mathrm{LiMn}_{2} \mathrm{O}_{4}(010)$

\subsection{2 (100)表面}

表 5 是 $\mathrm{LiMn}_{2} \mathrm{O}_{4}(100)$ 的表面能量表. $\mathrm{Li}$ 终端表面 $\mathrm{GGA}+\mathrm{U}$ 能量为 $0.654 \mathrm{~J} / \mathrm{m}^{2}$, 较相同方法计算所得 $\mathrm{Mn} / \mathrm{O}$ 的表面能 $1.195 \mathrm{~J} / \mathrm{m}^{2}$, 低约 $0.5 \mathrm{~J} / \mathrm{m}^{2}$. 主要考虑的是 $\mathrm{Li}$ 层 和 $\mathrm{Mn} / \mathrm{O}$ 层的形成能 $(\gamma)$, 其 $\gamma(\mathrm{Li}) / \gamma(\mathrm{Mn} / \mathrm{O}) \approx 1.5(\mathrm{GGA}+$ $\mathrm{U})$. 因此(100)表面中 $\mathrm{Li}$ 终端较为稳定的, 其原因是 $\mathrm{Li}$ 终端具有较少的断键空间结构.

表 $5 \mathrm{LiMn}_{2} \mathrm{O}_{4}(100)$ 的表面能量表(单位: $\mathrm{J} / \mathrm{m}^{2}$ )

Table 5 surface energy scale for $\mathrm{LiMn}_{2} \mathrm{O}_{4}(100)$ (unit: $\mathrm{J} / \mathrm{m}^{2}$ )

\begin{tabular}{cccc}
\hline Terminated & Surface energy & $\mathrm{Fu}^{[39]}$ & Benedek $^{[11]}$ \\
\hline Li terminated & 0.654 & 0.40 & 0.58 \\
$\mathrm{Mn} / \mathrm{O}$ terminated & 1.195 & 0.90 & 0.98 \\
\hline
\end{tabular}

\subsection{3 (110)表面}

表 6 是 $\mathrm{LiMn}_{2} \mathrm{O}_{4}(110)$ 的表面能量表. 计算出的

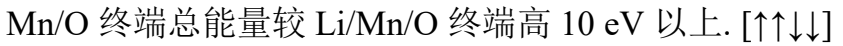
自旋排列 $\mathrm{Mn} / \mathrm{O}$ 终端表面能为 $1.050 \mathrm{~J} / \mathrm{m}^{2}$, 较 $\mathrm{Li} / \mathrm{Mn} / \mathrm{O}$ 终

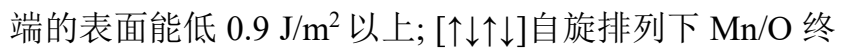
端表面能为 $1.061 \mathrm{~J} / \mathrm{m}^{2}$, 较 $\mathrm{Li} / \mathrm{Mn} / \mathrm{O}$ 终端的表面能也低 $0.9 \mathrm{~J} / \mathrm{m}^{2}$ 以上. 这表明在(110)表面情况下 $\mathrm{Mn} / \mathrm{O}$ 终端较 $\mathrm{Li} / \mathrm{Mn} / \mathrm{O}$ 表面稳定. 另外, 计算中发现(110)-AFM 型表 面在 $[\uparrow \uparrow \downarrow \downarrow]$ 自旋排列比 $[\uparrow \downarrow \uparrow \downarrow]$ 的自旋排列更加稳定. $\mathrm{Mn} / \mathrm{O}$ 终端比 $\mathrm{Li} / \mathrm{Mn} / \mathrm{O}$ 终端表面能小的原因可能是 $\mathrm{Mn} / \mathrm{O}$ 终端在表面含欠配位的锰原子. 这些表面锰原子 会与终端的氧形成 $\mathrm{MnO}_{6}$ 多面体, 降低配位缺陷致使稳 定性增强. 不同终止面间的键合性能会因不同的表面取 向而发生改变进而影响到 Mn 离子溶解 ${ }^{[37]}$.
表 $6 \mathrm{LiMn}_{2} \mathrm{O}_{4}(110)$ 的表面能量表(单位: $\mathrm{J} / \mathrm{m}^{2}$ )

Table 6 Surface energy scale for $\mathrm{LiMn}_{2} \mathrm{O}_{4}(110)$ (unit: $\mathrm{J} / \mathrm{m}^{2}$ )

\begin{tabular}{cccc}
\hline Terminated & Total energy & $\begin{array}{c}\text { Surface energy } \\
{[\uparrow \uparrow \downarrow \downarrow]}\end{array}$ & $\begin{array}{c}\text { Surface energy } \\
{[\uparrow \downarrow \uparrow \downarrow]}\end{array}$ \\
\hline Mn/O terminated & $\begin{array}{c}-364.243 \\
\mathrm{eV}\end{array}$ & 1.050 & 1.061 \\
$\mathrm{Li} / \mathrm{Mn} / \mathrm{O}$ terminated & -352.375 & 1.969 & 1.998 \\
& $\mathrm{eV}$ & & \\
\hline
\end{tabular}

\section{3 .4 (111)表面}

图 9(a)是未重构前(111)表面在三种情形下的表面 能，此时 $\mathrm{T} 1 、 \mathrm{~T} 2$ 有 18 层, T3 有 19 层. 增添额外 Mn 层 的 $\mathrm{T} 3$ 表面能增大到 $1.588 \mathrm{~J} / \mathrm{m}^{2}$. 比较 $\mathrm{T} 1 、 \mathrm{~T} 2 、 \mathrm{~T} 3$ 表面 能结果, 得出 $\mathrm{T} 2$ 情况下表面最稳定, 原因是 $\mathrm{T} 2$ 情形上 下层锂离子会与终端氧形成稳定的 $\mathrm{LiO}_{4}$ 四面体. $\mathrm{LiO}_{4}$ 四 面体的存在会促进电化学过程, 使锂电池材料具有更好 的高倍率性能 ${ }^{[40]}$.

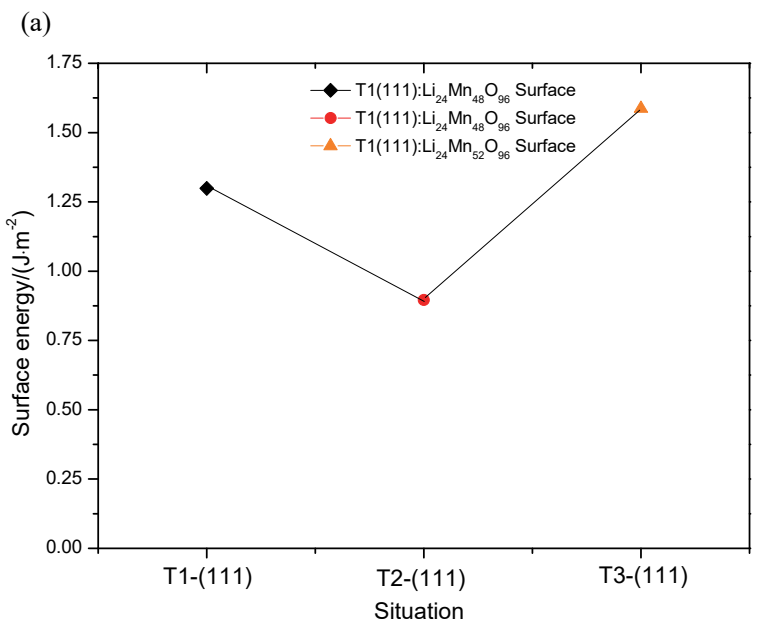

(b)

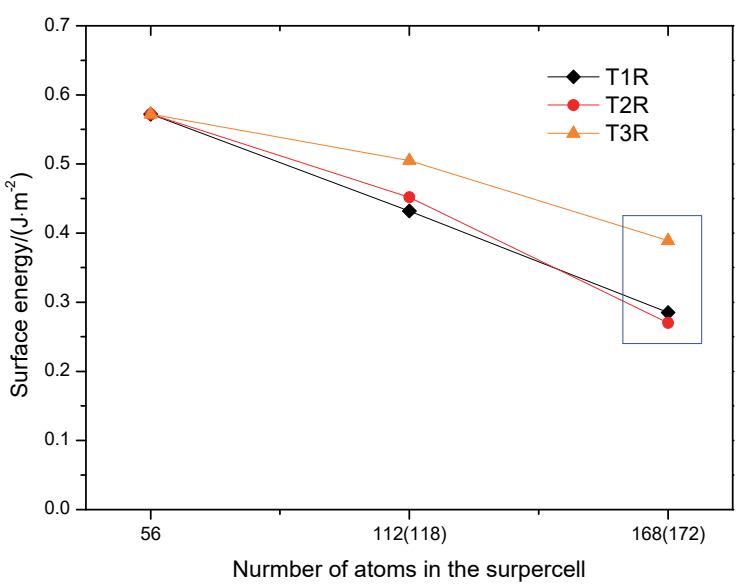

图 9 计算随着平板厚度增加的 DFT 表面能: (a) T1、T2 和 T3 的未构 建(111)表面能和(b)重构后表面能(T1R、T2R 和 T3R)

Figure 9 The surface energy of the DFT with the increase of plate thickness was calculated: (a) unconstructed (111) surface energy of T1, $\mathrm{T} 2$ and T3; (b) reconstructed surface energy (T1R, T2R and T3R)

图 9(b)是重构后的 T1R、T2R 和 T3R 表面能. 随着 板层的增加可以看到 $\mathrm{T} 1 \mathrm{R}$ 和 $\mathrm{T} 2 \mathrm{R}$ 的表面能大小接近，但 是 $\mathrm{T} 3 \mathrm{R}$ 的能量明显高于 $\mathrm{T} 1 \mathrm{R}$ 和 $\mathrm{T} 2 \mathrm{R}$. 这是由于 $\mathrm{T} 3 \mathrm{R}$ 终 
端属于非化学计量, 参考的体相能量化学组分处于基 态. 重构的(111) $\mathrm{LiMn}_{2} \mathrm{O}_{4} \mathrm{~T} 1$ 表面计算结果中, 较增加 板层后的 T1-2、T1-3 情况, T1-1 表面能最高, 达到 0.572 $\mathrm{J} / \mathrm{m}^{2}$. 图中折线走向显示出表面能随着板层增加逐渐降 低. 造成该结果的原因是表面的欠配位锰离子与底层区 域的单价锂离子交换位置会在表面附近形成反尖晶石 相, 当表面区域的锰配位数增加时, 反尖晶石重构会增 强结构的稳定性. 重构能够有效地在表面层产生部分反 尖晶石排列, 伴随着平均锰氧化态的降低. 这种终端重 构使(111)表面成为尖晶石结构中最稳定的切面.

\section{4 总结}

采用 $\mathrm{GGA}+\mathrm{U}$ 方法计算了 $\mathrm{LiMn}_{2} \mathrm{O}_{4}$ 晶体在不同的 化学计量比下的表面能量. 比较了 GGA 和 GGA+U 计 算 $\mathrm{LiMn}_{2} \mathrm{O}_{4}$ 晶体体相结构, 表明当 $\mathrm{Mn}$ 的 $\mathrm{d}$ 轨道选取有 效 $\mathrm{U}$ 值时晶格参数会明显变大. 使用相同的电子参数, 对(100)，(010)，(001)，(110)和(111)表面情况计算分析, 结果表明 $\mathrm{Li}$ 终端的(001)、(010)和(100)表面在缺锂条件 下能量较富锂条件下能量低. 而 $\mathrm{Mn} / \mathrm{O}$ 终端的(110)表面 较 $\mathrm{Li}$ 终端能量低. 关于反铁磁研究, (110)-AFM 型表面 在 $[\uparrow \uparrow \downarrow \downarrow]$ 自旋排列比 $[\uparrow \downarrow \uparrow \downarrow]$ 的自旋排列下更加稳定. 与 (001)、(010)、(100)、(001)表面的能量结果相比, 采用 选择性重构的(111)表面能最低. 当欠配位锰离子与底 层的锂离子交换会在表面附近形成稳定的反尖晶石相, 这使得(111)表面成为尖晶石结构中最稳定的切面. 除 (111)表面外, 其它低指数表面在铁磁和反铁磁下的表 面能相似. 研究结论为缓解 $\mathrm{LiMn}_{2} \mathrm{O}_{4}$ 材料容量衰减提供 理论依据, 为研究电化学条件下可能存在的新结构提供 理论参考.

\section{References}

[1] Cai, Z. F.; Ma, Y. Z.; Huang, X. N.; Yan, X. H.; Yu, Z. X.; Zhang, S. H.; Song, G. S.; Xu, Y. L.; Wen, C. E.; Yang, W. D. J. Energy Storage. 2020, 27, 101036.

[2] Lee, A.; Vrs, M.; Dose, W. M.; Niklas, J.; Johnson, C. S. Nat. Commun. 2019, 10, 977.

[3] Xu, G. J.; Liu, Z. H.; Zhang, C. J.; Cui, G. L.; Chen, L. Q. J. Mater. Chem. A 2015, 3, 4092.

[4] Liu, J.-D.; Zhang, Y.-D.; Liu, J.-X. Li, J.-H.; Qiu, X.-G.; Cheng, F.-Y. Acta Chim. Sinica 2020, 78, 1426 (in Chinese). (刘九鼎, 张宇 栋, 刘俊祥, 李金翰, 邱晓光, 程方益, 化学学报, 2020, 78, 1426.)

[5] Kozawa, T.; Harata, T.; Naito, M. J. Asian Ceram. Soc. 2020, 8, 309.

[6] Yu, Y.; Guo, J.; Xiang, M.; Su, C.; Duan, K. Sci. Rep. 2019, 1, 16864.
[7] Wei, T.; Zhuang, Q.-C.; Wu, C.; Cui, Y.-L.; Fang, L.; Sun, S.-G. Acta Chim. Sinica 2010, 68, 1481(in Chinese). (魏涛, 庄全超, 吴超, 崔 永丽, 方亮, 孙世刚, 化学学报, 2010,68, 1481.)

[8] Yu, F.; Zhang, J.-J.; Wang, C.-Y.; Yuan, J.; Yang, Y.-F.; Song, G.-Z Prog. Chem. 2010, 22, 9 (in Chinese). (于锋, 张敬杰, 王昌胤, 袁 静, 杨岩峰, 宋广智, 化学进展, 2010, 22,9.)

[9] Liu, Y. J.; Li, X. H.; Guo, H. J.; Wang, Z. X.; Hu, Q. Y.; Peng, W. J.; Yang, Y. Rare Metals 2009, 4, 322.

[10] Lin, C.; Robert, E. W.; Kan, S. C.; Joseph, A. L.; Christopher, J.; Zhen, Z. Y.; Mark, C.; Hersam.; Jeffrey, P.; Greeley.; Jeffrey, W. E. Chem 2018, 4, 2418.

[11] Benedek, R.; Thackeray, M. M. Phys. Rev. B 2011, 83, 173.

[12] Karim, A.; Fosse, S.; Persson, K. A. Phys. Rev. B 2013, 87, 178.

[13] Ouyang, C. Y.; Zeng, X. M.; Šljivancanin, Z.; Baldereschi, A. J. Phys. Chem. C 2010, 114, 4756.

[14] Wang, T.; Wang, W.; Zhu, D.; Duan, X.-B.; Wei, Z.-Q.; Chen, Y.-G. Chin. J. Inorg. Chem. 2014, 30, 2461 (in Chinese). (王婷, 王弯, 朱 丁，段晓波，魏治乾，陈云贵，无机化学学报, 2014, 30, 2461.)

[15] Bi, Z.; Zhao, N.; Ma, L.; Shi, C.; Guo, X. J. Mater. Chem. A 2020, 8, 4252 .

[16] Kresse, G.; Furthmüller, J. Comput. Mater. Sci. 1996, 6, 15.

[17] Kresse, G.; Furthmüller, J. Phys. Rev. B 1996, 54, 11169.

[18] Shi, S. Q.; Gao, J.; Liu, Y.; Zhao, Y.; Wu, Q.; Ju, W. W.; Ouyang, C.; Xiao, R. J. Chinese Phys. B 2016, 25, 018212.

[19] Wang, J.; Zhang, Z.; Zhang, Y. N.; Han, D.; Jin, L. L.; Sheng, L. Y.; Chartrand, P.; Medraj, M. Mater. Lett. 2019, 256, 126628.

[20] Saal, J. E.; Kirklin, S.; Aykol, M.; Meredig, B.; Wolverton, C. JOM 2013, 65, 1501

[21] Kirklin, S.; Meredig, B.; Wolverton, C. Adv. Energy Mater. 2013, 3, 252.

[22] Vallverdu, G.; Minvielle, M.; Andreu, N.; Gonbeau, D.; Baraille, I. Surf. Sci. 2016, 649, 46

[23] Shukla, A.; Gaur, N. K.; Ghosh, P. Appl. Surf. Sci. 2020, 527, 146703 .

[24] Ouyang, C. Y.; Shi, S. Q.; Lei, M. S. J. Alloys Compd. 2009, 474, 370.

[25] Tomeno, I.; Kasuya, Y.; Tsunoda, Y. Phys. Rev. B 2001, 64, 115.

[26] Singh, G.; Gupta, S. L.; Prasad, R.; Auluck, S.; Gupta, R.; Sil, A. J. Phys. Chem. Solids 2009, 70, 1200.

[27] Singh, P.; Sil, A.; Nath, M.; Ray, S. J. Electrochem. Soc. 2010, 157, 259.

[28] Lee, Y. K. Ph.D. Dissertation, The University of Michigan, United States, 2015.

[29] Garcia, J. C.; Bareo, J.; Chen, G.; Croy, J. R.; Iddir, H. Phys. Chem. Chem. Phys. 2020, 22, 24490.

[30] Yan, P.; Nie, A.; Zheng, J.; Zhou, Y.; Lu, D.; Zhang, X.; Xu, R.; Belharouak, I.; Zu, X.; Xiao, J. Nano Lett. 2015, 15, 514.

[31] Qian, K.; Tang, L. K.; Wagemaker, M.; He, Y. B.; Liu, D. Q.; Li, H.; Shi, R. Y.; Li, B. H.; Kang, F. Y. Adv. Sci. 2017, 4, 1700205.

[32] Xu, C.; Märker, K.; Lee, J. H.; Mahadevegowda, A.; Reeves, P. J.; Day, S. J.; Groh, M. F.; Emge, S. P.; Ducati, C.; Layla, M. B.; Tang, C. C.; Grey, C. P. Nat. Mater. 2020, $20,1$.

[33] Chen, J.; Wu, X. P.; Hope, M. A.; Qian, K.; Peng, L. Nat. Commun. 2019, 10, 5420 .

[34] Rasmussen, M. K.; Meinander, K.; Besenbacher, F.; Lauritsen, J. V. Beilstein J. Nanotechnol. 2012, 3, 192.

[35] Yoo, S. H.; Todorova, M.; Neugebauer, J. R. Phys. Rev. Lett. 2018, $120,066101$.

[36] Kim, S.; Aykol, M.; Wolverton, C. Phys. Rev. B 2015, 92, 115411.

[37] Fu, C. C.; Wang, J. Y.; Wang, J. F.; Meng, L. L.; Zhang, W. M.; Li, X. T.; Li, L. P. J. Mater Chem. A 2019, 7, 23149.

[38] Julien, C. M.; Zaghib, K. Electrochim. Acta 2004, 50, 411.

[39] Fu, K. M.S. Thesis, University of Pittsburgh, United States, 2014.

[40] Yu, F. D.; Wang, Z. B.; Chen, F.; Jin, W.; Zhang, X. G.; Gu, D. M. J. Power Sources 2014, 262, 104. 